\title{
日本・韓国鼻科学会セッション
}

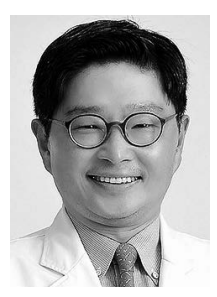

\section{ILC subsets in Asian nasal polyps}

\author{
Dong-Young Kim, MD, PhD \\ Department of Otorhinolaryngology-Head and Neck Surgery \\ Seoul National University College of Medicine
}

Chronic rhinosinusitis with nasal polyp (CRSwNP) is an umbrella term for a heterogeneous group of inflammatory disorders characterized by persistent polypoid inflammation of the sinonasal mucosa ( $>12$ weeks). There is a regional variation in CRSwNP endotypes: Western polyp shows a predominance of eosinophilic TH2-inflammation, whereas Asian polyp has a predominant neutrophilic infiltration and a local TH1/TH17 signature.

Innate lymphoid cell (ILC) is a group of innate immune cells that belong to the lymphoid lineage, but do not respond in an antigen-specific manner, as they lack a B or T cell receptor. ILCs have been shown to regulate defense against infection, adaptive immunity and wound healing through their prompt reactivity to tissue infection and injury. ILCs can be categorized according to their cytokine secretion. It mirrors the phenotypes and function of $\mathrm{CD}^{+} \mathrm{T}$ helper cells. CRSwNP and eosinophilic CRS have been linked with a skewed Th2 immune response through eosinophilic inflammation mainly mediated through IL-5 and IL-13 cytokines. The discovery of ILC2s, which promote Th2 inflammation, represents an important finding in immunology and highlights a potential role within CRS. It was reported that the frequency of ILC2s were 2.23 times enriched in western nasal polyps compare to controls. However, the study on ILC subsets in Asian nasal polyps is still lacking.

We aimed to analyze ILC subsets in CRS tissue across different phenotypes (CRSwNP / CRSsNP) and endotypes (eosinophilic CRS / non-eosinophilic CRS) in Asian patients, and correlation between clinical characteristics such as serum total IgE, blood eosinophils, and CT score and the frequency of ILC subsets. The effect of allergic rhinitis (AR) on ILC subsets in NP tissue was also evaluated.

Samples were collected from both uncinate tissue and nasal polyps of patients with CRSwNP, and from uncinate tissue of patients with CRS without nasal polyp (CRSsNP) and normal control. Single cell suspension was achieved, and then ILC subtypes were analyzed using flow cytometry. Real-time PCR was performed to analyze the mRNA expression levels of major ILC-activating cytokines.

Sixty percent of CRSwNP patients had eosinophilic NP, while most uncinate tissues of CRSsNP were non-eosinophilic (93.7\%). ILC2 frequency was significantly higher in NP and eosinophilic CRS (ECRS). NCR (natural cytotoxicity receptor)- ILC3 was also increased in NP and ECRS. IL-22-producing ILC3s were elevated in NP of CRSwNP. ILC percentage was not significantly correlated with serum total IgE, blood eosinophil count, and Lund-Mackay CT score. Allergic rhinitis increased ILC2 in NP tissues, but not NCR- ILC3. Therefore, NCR ${ }^{-}$ILC3s as well as ILC2s may contribute to the pathogenesis of nasal polyp in Asian CRSwNP patients.

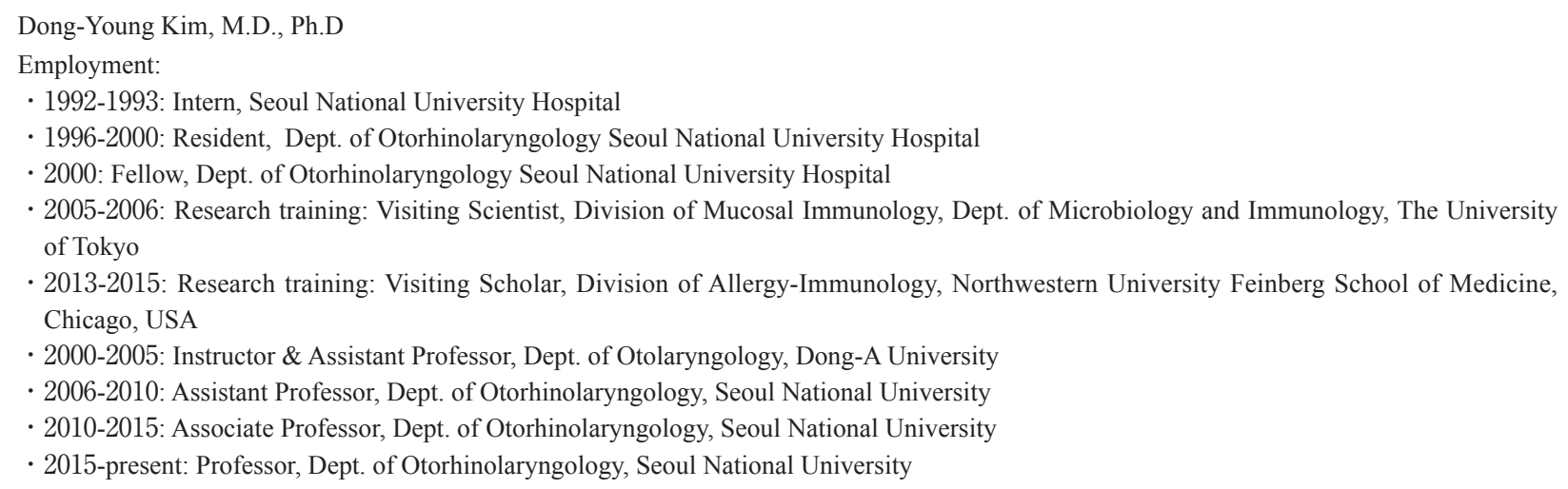




\title{
日本・韓国鼻科学会セッション
}

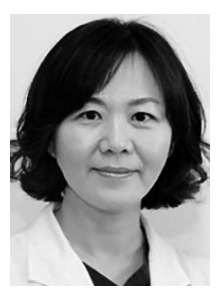

\section{Olfactory Training for Patients with Olfactory Disorder}

\author{
Mi Kyung Ye
}

Department of Otolaryngology, School of Medicine, Catholic University of Daegu

Olfactory dysfunction affects a significant percentage of the general population, estimated at around $15 \%$. An acquired loss of olfactory function has a major impact on the quality of life.

To date there is no validated pharmacotherapy for olfactory dysfunction, although attempts have been made to establish such treatment including trials with systemic and topical steroids, vitamin B, caroverine, alpha-lipoic acid, minocycline, or acupuncture.

Olfactory Training (OT) is an alternative treatment based on modulation of the regeneration process of the olfactory system via repeated exposure to odors. It was described by T. Hummel and the idea was based on Henning's work on primary odors and basic odor categories.

We conducted a prospective study of the impact of olfactory training in patients with postinfectious, post-traumatic and idiopathic olfactory dysfunction. For their training, patients exposed themselves to four different odors (phenylethyl alcohol: rose odor, eucalyptol: eucalyptus odor, citronellal:lemon odor, and eugenol: cloves odor) twice a day. Olfactory function was evaluated at baseline and again at 3, 6,9 months, and results were quantified in the form of each patient's TDI (threshold, discrimination, and identification) score.

The etiology of olfactory disorder and sex had statistically significant influence on the improvement rate of olfactory function. However, initial severity and duration of olfactory loss had no statistically significant influence on the improvement rates after OT. The TDI score increased primarily because of improvements in the discrimination and identification components. In this presentation, our treatment outcomes will be shown on the basis of our data and the effects of OT in patients with olfactory dysfunction will be discussed.

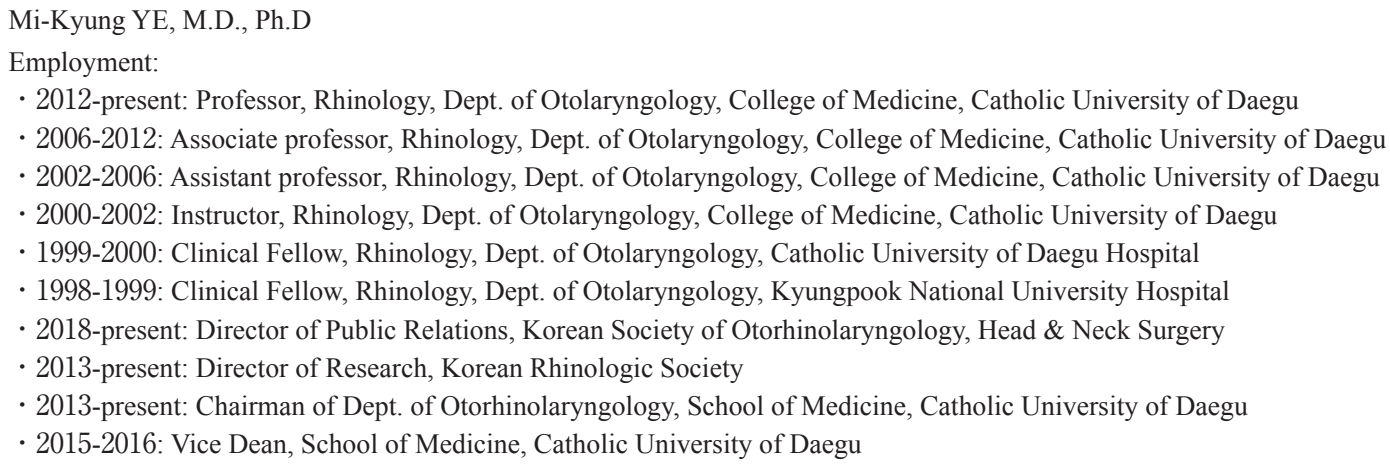




\title{
日本・韓国鼻科学会セッション
}

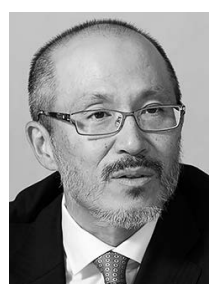

\section{Effects of subcutaneous immunotherapy (SCIT) on the numbers of IL-10-producing CD4 ${ }^{+} \mathrm{T}$ cells} and IL-10-producing $B$ cells in peripheral blood of pollinosis patients

\author{
Tetsuya Terada ${ }^{1}$, Yusuke Ayani ${ }^{1}$, Tatsurou Kuriyama ${ }^{1}$, Takuya Yoshida ${ }^{1}$, Norio Suzuki $^{1}$, Yuko Inaka ${ }^{1}$, \\ Takaki Inui ${ }^{1}$, Masaya Matsuda ${ }^{2}$, Takeshi Nabe ${ }^{2}$, Ryo Kawata ${ }^{1}$ \\ ${ }^{1}$ Osaka Medical College, Takatsuki, Japan \\ ${ }^{2}$ Setsunan University Department of Immunopharmacology, Faculty of Pharmaceutical Sciences, \\ Hirakata, Japan
}

\section{Introduction:}

It has been suggested that mechanisms underlying effectiveness of antigen-specific immunotherapy are related to increases in IgG4 antibody and regulatory $\mathrm{T}$ and $\mathrm{B}$ cells, whereas their precise roles have been unclear.

\section{Method:}

After the pollen season in 2017, peripheral blood was drawn from normal subjects, Japanese cedar plollinosis patients and patients treated with SCIT for more than 3 years. Peripheral blood mononuclear cells (PBMC) were collected by a density gradient method, and stimulated with the specific antigen, Cry $\mathrm{j} 1$ or anti-CD3/CD28 mAb. Then, cell surface molecules and intracellular IL10 and Foxp3 were stained, followed by flowcytometer analyses.

\section{Results:}

1) Although increase in antigen-specific IgE antibody level was not statistically reduced by SCIT, antigen-specific IgG4 level was dramatically increased in the SCIT-treated patients. 2) The number of antigen-induced IL-10-producing CD4 $4^{+} \mathrm{T}$ cells in PBMC of the pollinosis patients was significantly lower than that of the normal subjects. However, the number of antigen-induced IL-10producing $\mathrm{CD}^{+} \mathrm{T}$ cells of the SCIT-treated pollinosis patients was comparable to that of the normal subjects. Most of the IL-10producing $\mathrm{CD}^{+} \mathrm{T}$ cells were negative for Foxp3, a transcription factor of naturally occurring regulatory $\mathrm{T}$ cells. 3) The number of IL-10-producing B cells of the pollenosis patients was not different from that of normal subjects, whereas that of SCIT-treated patients was significantly higher than those of other 2 groups.

\section{Conclusion:}

Regulatory functions of the IL-10-producing CD4+ Foxp3- T cells and IL-10-producing B cells could be involved in mechanisms underlying the clinical effects of SCIT.

E-mail: oto056@osaka-med.ac.jp

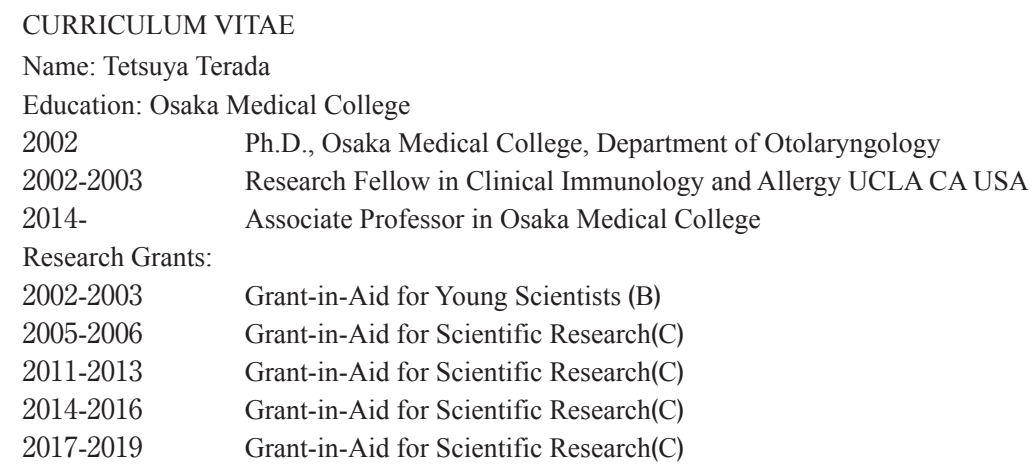




\title{
日本・韓国鼻科学会セッション
}

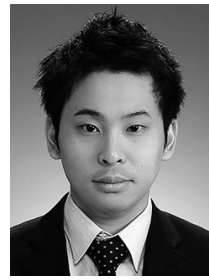

Detection of local IgE in Eosinophilic and non-Eosinophilic Rhinosinusitis

\author{
Nozomu Wakayama \\ Department of Otolaryngology \\ Nippon Medical School, Musashikosugi Hospital, Kanagawa, JAPAN
}

Eosinophilic chronic rhinosinusitis (ECRS) has been known as a very intractable and recurrent type chronic rhinosinusitis with severe eosinophil infiltration in sinus mucosa and/or polyps, complicated often with asthma. The most sensitive inflammatory sites are known as the ethmoid sinuses bilaterally among all the paranasal sinuses. Recently, the diagnostic standard of ECRS was established in Japan. ECRS ware registered as one of the intractable diseases of the Japan national health care system in 2015. However, detailed pathophysiology of ECRS is still under debate. The peripheral blood total IgE/antigen-specific IgE(sIgE) levels was not related ECRS. According to previous reports, however, total $\operatorname{IgE}$ and $\mathrm{sIgE}$ in nasal polyps are related to local eosinophilic inflammation. And the Omalizumab (Anti-human IgE monoclonal antibody) demonstrated clinical efficacy in the treatment of nasal polyps with eosinophil accumulation. Which motivated us to do this study as follows. In order to analyze the total Ig E and antigenspecific IgE (sIgE) in ethmoid sinus mucosa in ECRS-pathology, harvested ethmoid sinus mucosa during operation was processed to estimate the mucosal total $\operatorname{IgE}$ and $\operatorname{sgE}$ (enterotoxin, fungi, pollen and mites) per gram, per milliliter phosphate buffer saline, followed by comparison between ECRS and non-ECRS groups. As the results, mucosal total IgE level was statistically higher in ECRS than in NECRS, while there was no significant difference in each sIgE level in these two groups. Other related data are additionally to be presented with the discussion.

\footnotetext{
Nozomu Wakayama, M.D., Ph.D

Education:

- 2009: M.D., Iwate Medical University, School of Medicine

- 2017: Ph.D., Graduate from Nippon Medical School, Graduate School of Medical Science

Employment:

- 2009-2011: Clinical trainee, Saiseikai Central Hospital

- 2011-2013: Resident, Dept. of Otorhinolaryngology, Head and Neck Surgery, Nippon Medical School

- 2013-2017: Research Associate Dept. of Otolaryngology Nippon Medical School, Musashikosugi Hospital

- 2017-present: Assistant Professor Dept. of Otolaryngology Nippon Medical School, Musashikosugi Hospital
} 